\title{
DIABETES PRÉ-GESTACIONAL: EXPERIÊNCIA DE GRÁVIDAS COM O CONTROLE DA DOENÇA*
}

Aliny de Lima Santos', Elen Ferraz Teston', Hellen Pollyanna Mantelo Cecilio², Deise Serafim³, Sonia Silva Marcon ${ }^{4}$

${ }^{1}$ Enfermeira. Doutoranda em Enfermagem. Universidade Estadual de Maringá. Maringá-PR-Brasil.

${ }^{2}$ Enfermeira. Mestre em Enfermagem. Universidade Estadual de Maringá. Maringá-PR-Brasil.

${ }^{3}$ Enfermeira. Doutora em Enfermagem. Universidade Estadual de Maringá. Maringá-PR-Brasil.

${ }^{4}$ Enfermeira. Doutora em Filosofia da Enfermagem. Universidade Estadual de Maringá. Maringá-PR-Brasil.

RESUMO: Estudo descritivo de natureza qualitativa, realizado com o objetivo de conhecer como gestantes com diabetes pré-existente vivenciam o cuidado com a doença. As informantes foram cinco mulheres acompanhadas no Ambulatório de Pré-natal de alto risco em um Hospital Universitário. Os dados foram coletados em junho e julho de 2010, por meio de entrevistas semi-estruturadas, realizadas no ambulatório, na consulta pré-natal que foram gravadas, transcritas integralmente e submetidas à análise de conteúdo, modalidade temática. Emergiram duas categorias: Mudanças a partir da gestação estimuladas pela família, e Reconhecendo a importância do acompanhamento profissional e suas orientações, as quais mostram a implementação de mudanças significativas no autocuidado sendo a gravidez e as orientações profissionais apontadas como fatores motivadores para as mesmas. Conclui-se que a gestação, além de constituir um marco na vida das mulheres, também atuou como fator modificador da percepção e adesão ao autocuidado.

DESCRITORES: Diabetes mellitus tipo 2; Gestantes; Autocuidado; Cuidados de enfermagem.

\section{PRE-GESTATIONAL DIABETES: PREGNANT WOMEN'S EXPERIENCE WITH DISEASE CONTROL}

\begin{abstract}
A descriptive qualitative study, carried out in order to know how pregnant women with pre-existing diabetes deal with the illness. The informants were five women in a high risk prenatal clinic of a University Hospital. The data were collected in June and July 2010, through semi-structured interviews that were recorded, transcribed and analyzed for thematic content, conducted during clinic and prenatal consultations. Two categories emerged: changes encouraged by family members after pregnancy and recognizing the importance of professional monitoring and its guidelines, which show the implementation of significant changes in self-care, with pregnancy and professional guidelines cited as motivating factors. This shows that pregnancy, besides being a landmark in women's lives, also acts as a modifying factor in perception and self-care.
\end{abstract}

DESCRIPTORS: Diabetes mellitus type 2; Pregnant women; Self-care; Nursing care.

\section{DIABETES PREGESTACIONAL: EXPERIENCIA DE GRÁVIDAS CON EL CONTROL DE LA ENFERMEDAD}

RESUMEN: Estudio descriptivo de naturaleza cualitativa, realizado con el objetivo de saber cómo gestantes con diabetes ya existente viven el cuidado con la enfermedad. Las participantes fueron cinco mujeres acompañadas en el Ambulatorio de Prenatal de alto riesgo en un Hospital Universitario. Los datos fueron obtenidos en junio y julio de 2010, por medio de entrevistas semiestructuradas, realizadas en el ambulatorio, en las consultas de prenatal que fueron grabadas, transcriptas integralmente y sometidas al análisis de contenido, modalidad temática. Resultaron dos categorías: Cambios a partir de la gestación estimuladas por la familia, y Reconociendo la importancia del acompañamiento profesional y sus orientaciones, las cuales muestran la implementación de cambios significativos en el autocuidado, siendo la gravidez y las orientaciones profesionales apuntadas como factores motivadores para estas. Se concluye que la gestación, además de constituir un mojón en la vida de las mujeres, también ha actuado como factor modificador de la percepción y adhesión al autocuidado. DESCRIPTORES: Diabetes mellitus tipo 2; Gestantes; Autocuidado; Cuidados de enfermería.

*Artigo oriundo da disciplina Os sistemas de cuidar/cuidado humano do Programa de Pós-Graduação em Enfermagem. Universidade Estadual de Maringá, 2011 


\section{INTRODUÇÃO}

O Diabetes Mellitus (DM) durante a gestação pode repercutir na saúde materna, fetal e perinatal. Contudo, quando preexistente à gravidez está associada a resultados obstétricos e perinatais desfavoráveis ${ }^{(1-2)}$. A avaliação do desempenho metabólico, bem como o controle eficiente desde o período que antecede a gestação é fundamental para um melhor prognóstico obstétrico. O controle metabólico ineficiente no período da organogênese fetal está associado ao aumento da taxa de abortamento espontâneo e de malformações fetais, aumentando o risco de macrossomia e suas comorbidades associadas ${ }^{(3)}$.

Complicações associadas à doença, a exemplo da vasculopatia, contribuem ainda para os maus resultados obstétricos como o aumento do risco de parto pré-termo, restrição do crescimento fetal, doença hipertensiva da gravidez e morbimortalidade perinatal ${ }^{(2-3)}$.

Na prática assistencial, deparamo-nos com o impacto que o DM tem sobre a saúde da população de modo geral e com a dificuldade de adesão a comportamentos saudáveis que fazem parte do controle da doença ${ }^{(4)}$. Independente do sexo, faixa etária ou tempo de diagnóstico, o maior desafio para o controle é manter a glicemia dentro de parâmetros adequados, demandando mudanças nos hábitos de vida, com vistas ao uso correto de antidiabéticos, mudanças dos hábitos alimentares, prática regular de atividades físicas periódicas, além de conscientização sobre sua situação de saúde ${ }^{(5)}$.

O cuidado com a doença como meio de controle dos níveis glicêmicos de grávidas com diabetes pré-existente torna-se ainda mais importante, já que este constitui um aspecto terapêutico fundamental para prevenir as complicações e morbidades geralmente associadas aos casos mal controlados ${ }^{(4)}$. Fazse então necessário conhecer como se dá a convivência destas grávidas com a doença e o seu cuidado, no intuito de subsidiar a assistência prestada pela equipe de saúde, a fim de obter maior êxito na evolução da gravidez, que nesse caso é considerada como gestação de risco ${ }^{(6)}$.

Dada à importância do tema, tanto para a prevenção de complicações gestacionais, do parto e puerpério, quanto para a qualificação da prática assistencial clínica, o DM gestacional tem sido amplamente discutido em seus aspectos fisiopatológicos $^{(6-7)}$. Sendo assim, o estudo teve como objetivo conhecer como grávidas com diabetes pré-gestacional vivenciam a doença e seu cuidado.

\section{METODOLOGIA}

Estudo descritivo de natureza qualitativa, realizado em uma cidade do noroeste do Paraná, junto a cinco grávidas com DM tipo 2, acompanhadas no Ambulatório de Pré-Natal de Alto Risco de um Hospital Universitário. Este ambulatório é referencia para 30 cidades que integram a $15^{\mathrm{a}}$ Regional de Saúde do Paraná, funciona diariamente no período da manhã e tarde, realizando em média 20 atendimentos à gravidez de risco por dia.

Os dados foram coletados nos meses de junho e julho de 2010, por meio de entrevistas semiestruturadas realizadas antes ou após as consultas, em sala reservada no próprio ambulatório. A solicitação de participação no estudo foi feita pessoalmente a cada mulher, após identificar as gestantes que tinham diabetes pré-existente, que foi o critério de inclusão. O instrumento utilizado na entrevista foi constituído de duas partes: uma com questões relacionadas aos dados socioeconômicos e clínicos, e outra composta pelas seguintes perguntas norteadoras: Para você, como é conviver com o diabetes? Faleme sobre o cuidado que você tem com a doença. Você considera que esse cuidado mudou com a gestação? Como e por quê?

Após o consentimento, as entrevistas foram gravadas com uso de aparelho eletrônico, o que permitiu maior interação entre entrevistador/ entrevistado, sendo transcritas integralmente e submetidas à análise de conteúdo modalidade temática composta por três fases: a pré-análise, a exploração do material e o tratamento dos dados $^{(8)}$. A pré-análise é a fase de organização dos documentos em que ocorreram a leitura flutuante, a escolha dos relatos, a formulação de hipóteses e a elaboração de indicadores para fundamentar a interpretação. A etapa de exploração do material consistiu em encontrar agrupamentos e associações que respondessem aos objetivos do estudo surgindo, assim, as categorias. Já, a fase 
de tratamento dos resultados compreendeu o momento em que foram realizadas as inferências e a interpretação dos resultados encontrados.

O desenvolvimento do estudo ocorreu em conformidade com o preconizado pela Resolução do Conselho Nacional de Saúde, que normatiza as pesquisas com seres humanos, sendo aprovado pelo Comitê Permanente de Ética em Pesquisa com Seres Humanos (COPEP) da Universidade Estadual de Maringá (Parecer n. 215/2010). Todas as participantes assinaram o Termo de Consentimento Livre e Esclarecido e estão identificadas com nomes de flores.

\section{RESULTADOS}

Caracterizar as cinco participantes da pesquisa foi importante, pois o conhecimento do contexto familiar e histórico clínico permitiu maior compreensão do sentido que o autocuidado e o DM têm em suas vidas.

Margarida tinha 24 anos, primigesta, estava na $31^{\text {a }}$ semana gestacional e desenvolveu Doença Hipertensiva da Gravidez (DHG). Diagnóstico de DM há dois anos e fazia uso diário de Insulina (15U 1x/dia). Era do lar, possuía segundo grau completo e renda familiar de dois a três salários mínimos. Seu grupo familiar era composto por ela, marido e duas enteadas.

Jasmim tinha 38 anos, primigesta, estava na $29^{a}$ semana gestacional e desenvolveu DHG. Tinha diagnóstico de DM há quatro anos e realizava tratamento não farmacológico. Profissional de educação física tinha renda familiar de três salários mínimos. Seu grupo familiar era composto por ela e o marido.

Rosa tinha 37 anos, primigesta, estava na $23^{\text {a }}$ semana gestacional. Hipertensa, tinha diagnóstico de DM há sete anos e fazia uso de antidiabético oral (um comprimido 12/12h). Do lar, possuía segundo grau completo e renda familiar de um salário mínimo. Seu grupo familiar era composto por ela, marido, mãe e irmão.

Miosótis tinha 35 anos, primigesta, estava na $39^{\text {a }}$ semana gestacional. Hipertensa, tinha diagnóstico de DM há quatro anos e realizava tratamento não farmacológico. Era do lar, possuía segundo grau completo e renda familiar mensal familiar de dois salários mínimo. Seu grupo familiar era composto por ela, marido e enteada.

Orquídea tinha 44 anos, multigesta e estava na $40^{\mathrm{a}}$ semana gestacional. Tinha diagnóstico de DM há dois anos e seis meses e realiza tratamento não farmacológico. Era doméstica, possuía segundo grau incompleto e renda familiar de três salários mínimo. Seu grupo familiar era composto por ela, marido e dois filhos.

Mediante análise sistemática dos depoimentos emergiram duas categorias, conforme segue.

\section{Mudanças motivadas pela gestação e estimuladas pela família}

A doença e a gestação acarretaram mudanças significativas na relação com o próprio corpo e com aspectos do cotidiano, como por exemplo, a realização do controle glicêmico.

Eu não fazia [controle do DM] de jeito nenhum. Media quando ia ao clínico geral e quando ele pedia exames. Ah, Eu só ia a cada cinco meses. Um relaxo mesmo. Agora, estou medindo todos os dias, duas vezes por dia, melhorou muito. (Jasmim)

Olha, eu não ia ao médico, e quando ia, era de três em três meses, não me preocupava muito não. Medir a diabetes mesmo, eu só media de vez em quando. Agora mudou, vou ao médico todo mês, e estou medindo o diabetes todos os dias, estou tendo mais cuidado. (Rosa)

Eu estou fazendo o exame do dedinho [glicemia capilar] sempre, por que antes eu só fazia quando ia ao médico do posto, de três em três meses. Agora eu vejo quase todo dia. (Orquídea)

Mudanças também foram observadas nos hábitos alimentares, não apenas por ocasião do diagnóstico do DM, mas sim, tendo a gestação como principal motivador.

Eu acordava de manhã, tomava café com leite e comia 2 pães. Ai quando dava fome, que eu não tinha horário, eu ia lá e almoçava e comia uma esfirrinha, uma coxinha, não é? Ah! Mudou $100 \%$, eu acordo de manhã, tomo meia xícara de leite desnatado, com café e adoçante e três bolachas de sal. Dez horas uma fruta. Meio dia em ponto eu almoço arroz, legumes, salada, 
um pedaço de carne, cozida de preferência. (Margarida)

Eu sempre preferia salgado frito para variar, não é? Agora, está diferente, não estou mais comendo essas coisas, me alimento melhor, como salada todos os dias, como fruta, tomo leite, estou fazendo bem direitinho agora. E é bom! A gente se sente mais saudável. (Jasmim)

No depoimento de Rosa, percebe-se o reconhecimento de um hábito alimentar inadequado, mas também a dificuldade de modificá-lo.

Ah, quase que não mudou porque esses dias eu fiz polenta, comi logo dois pratos e não pode comer isso muito não é? E aquele miojo? Eu como quase todo dia. Eu como pão, bolo. Não tem jeito viu, você não pode, mas quando vai ver, já está comendo. (Rosa)

Só teve uma coisa que eu não consegui mudar muito ainda, foram as comidas, por que eu como o que tem não é?! Sei que não tá certo comer pão e mortadela todo dia, mas é o que tem, daí eu como. (Orquídea)

Mudanças na prática de atividades físicas também foram identificadas, como é possível observar na fala de Jasmim e Margarida.

Não fazia exercício por que não tinha tempo. Saia do serviço, que era bem corrido, e ia andando pra casa, daí já achava que era caminhada. Agora que não estou mais trabalhando, saio caminhar nem que seja duas vezes por semana. (Rosa)

Caminhada eu realmente fazia. Agora esse mês eu já não aguento fazer mesmo. Eu ando dentro de casa para não ficar parada, mas eu não consigo mais fazer caminhada. (Margarida)

Percebe-se, que a gestação traz consigo a responsabilidade materna, e assim a preocupação com o bem estar do filho constitui um marco para início e implementação de mudanças.
Eu vivo por ele [o filho], e para deixar que ele cresça. É uma responsabilidade única e exclusivamente minha. Se eu não me preocupar com ele, quem vai se preocupar? Por isso que eu me cuido, cuido da minha alimentação, para viver para ele. (Margarida)

A vida mudou, a cabeça mudou, tudo mudou. E hoje em dia eu sei que se eu me entupir de doce, eu não vou conseguir nada, a não ser me prejudicar e prejudicar a ele [o filho] que precisa de mim. Então, é isso que mudou. Eu preciso me cuidar, por causa dele. (Jasmim)

As gestantes passaram a ter como objetivo de vida o bom controle metabólico e consequentemente melhor qualidade de vida, no intuito de preservar-se saudável para o filho que aguardam.

Pretendo depois que eu ganhar ele [o filho], emagrecer um monte também para eu poder estar mais tempo junto com ele. (Margarida)

Agora é diferente, eu tenho ela [a filha] não é? Tenho que controlar a alimentação mais ainda, fazer caminhada, cuidar de mim, para poder cuidar dela, por que ela só tem a mim não é? Eu sou a mãe dela. (Miosótis)

Contudo, uma das maiores ferramentas para que o controle alimentar seja facilitado, é a participação, apoio e incentivo da família.

A minha vida e a do meu marido mudou. Ele come igual a mim [se referindo à adesão a hábitos de alimentação saudáveis]. Porque antes, ele trazia bomba de chocolate, bolo, paçoquinha e hoje em dia ele não traz mais. Ele diz que não é bom para mim, nem para o bebê. (Margarida)

Minha prima, o dia que eu dou uma mudadinha, como um pedacinho de pão, ela pega no meu pé, diz assim: 'Para por aí, que não é assim. Você nem pode comer isso'. O meu marido também controla a minha alimentação agora, nada de doce em casa. Está todo mundo ali junto, na luta. (Jasmim) 
Quando a mudança da rotina alimentar não abrange a família como um todo, pode constituir fator negativo referente à adesão da gestante a uma alimentação saudável.

Eu tento não comer, mais eu faço a comidinha pra eles [familiares] e fico com preguiça de fazer outra pra mim, aí eu como o mesmo que faço para eles. Como carne frita, batata frita, essas coisas. Eu sei que não posso comer, mais eles comem, daí eu fico com vontade. (Rosa)

\section{Acompanhamento profissional e orientações}

Identificou-se que o relacionamento entre o profissional de saúde e o cliente é fundamental na orientação das práticas de cuidado e na implementação das mesmas.

Antes eu não tinha acompanhamento com nutricionista, endocrinologista, o médico não estava o tempo todo falando e me dizendo como eu tinha que fazer. Eu converso sempre com a enfermeira. E toda hora a gente fica conversando, ai ela me falava: 'Calma, não é assim. Você pode ter uma vida normal'. (Margarida)

Lá no postinho que eu ia antes pra consultar do diabetes, já era muito bom sabe? Por que as meninas [enfermeiras] me falavam as coisas que eu tinha que fazer. Mas, aqui com as meninas daqui, com o jeitinho que elas tem pra falar, parece que ficou melhor, eu consigo controlar melhor. (Orquídea)

Entretanto, o modo como se ensina pode ser insuficiente para que ele associe o cuidado às suas atividades diárias.

As orientações foram boas também. Porque antes, como eu não ia ao médico, e eu não procurava saber o que eu tinha que fazer, o que eu tinha que comer, como é que eu tinha que me tratar. Eu vivia do jeito que eu queria. Hoje em dia, eu sei como é que eu preciso viver para viver bem. Eu tenho as meninas aqui [enfermeiras], a gente fez amizade. Ai uma já vem: 'E ai, controlou? Está controlando?' (Jasmim)
Aqui, com a assistência que me dão, parece que eu me sinto mais bem cuidado, por que eles se preocupam mesmo com a gente, eles estão sempre perguntando como a gente está e se não vem na consulta, eles ligam em casa pra saber o que aconteceu. Agora parece que até entendo melhor a doença, por que eles conversam comigo de um jeito que eu entendo. (Miosótis)

\section{DISCUSSÃO}

Ao descobrirem-se grávidas e receberem as orientações sobre o cuidado referente ao DM e as complicações que o mesmo pode acarretar para o feto, percebeu-se que todas as gestantes relataram mudanças de comportamento. Tal realidade também é percebida em outros estudos, os quais afirmam que as orientações compartilhadas às pacientes, modificam não somente o nível de conhecimento que elas possuem sobre DM e o cuidado, mas vão além, alterando suas práticas diárias, ou seja, o conhecimento teórico é transformado em prática de cuidado $^{(9-10)}$.

Sabe-se que a adesão a hábitos alimentares saudáveis, constitui ferramenta imprescindível para o controle glicêmico trazendo benefícios à saúde materno-fetal, e quando estas são aceitas de forma mais natural, elas tendem a ser adotadas com mais tranquilidade ${ }^{(11)}$. Nos depoimentos de Margarida e Jasmim, verificou-se que essa aceitação foi motivada pela gravidez. Entretanto, é necessária a conscientização da necessidade de controle alimentar não somente no período gestacional, já que o DM constitui uma patologia crônica que quando não controlado pode desencadear complicações ${ }^{(2,12)}$.

O manejo eficaz do diabetes na gestação depende da adesão da mulher ao plano de cuidados, que tem por objetivo manter a glicemia controlada. No entanto, este manejo não é algo fácil de ser alcançado, pois hábitos alimentares são formados ao longo da vida e assim de difícil modificação.

É muito importante compreender os riscos de manter uma alimentação inadequada, considerando este foi o primeiro passo para uma mudança de hábitos; porém não é suficiente. Nesses casos, acredita-se que os profissionais de saúde devem buscar reconhecer dificuldades vivenciadas pela mulher grávida em adotar 
práticas saudáveis, favorecendo melhor controle glicêmico, por meio de mudanças de hábitos de forma consciente e mais palpável possível ${ }^{(13)}$. Salienta-se ainda, que a gravidez é um momento impar na vida da mulher, novos plano de futuro é formulado, devendo, portanto, ser aproveitada como oportunidade para a manutenção preventiva da saúde e assim para a educação para a saúde ${ }^{(14)}$.

Assim como a necessidade de alteração de hábitos alimentares, mulheres com diabetes, sem complicações obstétricas e clínicas, devem ser encorajadas a iniciar ou continuar um programa de exercício moderado, como parte do tratamento e prevenção das complicações da doença. Estas provocam aumento da sensibilidade dos tecidos à insulina, melhoram o controle glicêmico, tendo efeito diurético e contribuindo para uma maior auto-estima $^{(15)}$.

A concepção de saúde e o modo como cada pessoa enfrenta a doença são desenvolvidos a partir das experiências pessoais e, essas guardam relação direta com crenças e valores os quais são formados ao longo da vida. No entanto, alguns fatores e eventos podem influenciar positivamente nesse enfrentamento e na mudança de condutas. Observou-se, nos depoimentos de Margarida e Jasmim, que a gestação foi capaz de motivar a adesão a práticas saudáveis, seja ela em maior ou menor escala, além de certo planejamento para o futuro, tendo o filho ${ }^{(a)}$ como motivação para as mudanças de atitudes que beneficiariam a sua saúde, frente sua responsabilidade de mãe.

Apesar de vivenciarem uma doença de difícil adaptação como o diabetes, o nascimento de um bebê saudável e o desejo de estar presente de forma ativa e saudável durante seu crescimento é sempre uma preocupação manifestada pelas grávidas ${ }^{(6)}$.

Mudanças nas práticas de cuidado, especialmente no que diz respeito à rotina alimentar, são determinadas no convívio familiar e social, sendo que é importante que todos colaborem para tornar os hábitos de vida e alimentares da gestante mais adequados, da forma mais facilitada possível ${ }^{(7)}$. A modificação da dieta e a proposta de formação de novos hábitos alimentares devem respeitar as preferências dos indivíduos, sua cultura e meio em que está inserido.

Percebe-se nos depoimentos que para o fortalecimento do controle da doença e assim a melhor convivência com a mesma, o apoio familiar é fundamental. Este se dá por meio da modificação de hábitos de todos os membros, apoiando a gestante e ainda melhorando a saúde de toda a famíliaa ${ }^{(16)}$. O potencial da participação familiar nos cuidados com a doença deve ser valorizado pelos profissionais da saúde de modo a melhorar o prognóstico da doença, sendo usada por tanto, como ferramenta fundamental no controle e manutenção da mesma ${ }^{(17)}$.

Em contrapartida, quando o apoio familiar, por meio da mudança da rotina alimentar não ocorre, torna-se necessária a adoção de uma alimentação diferenciada por parte da gestante, necessitando preparar uma refeição para a família e outro para si. Isto pode ser uma barreira na adesão à terapia nutricional, pois frente a esta situação, não é incomum que a mulher com diabetes não se sinta motivada para alimentar-se adequadamente, ou seja, de forma diferenciada dos demais, e, por conseguinte, não seguindo as recomendações necessárias ao seu tratamento, como se evidenciou no depoimento de Rosa.

Para alguns autores, a família tem sob sua responsabilidade atividades importantes, tais como cuidar de seus membros, limpeza doméstica, seleção e preparação dos alimentos, enfim, os aspectos envolvidos no apoio à saúde $^{(18)}$. Na presença de doenças como o DM, os familiares atuam basicamente como cuidadores. As ligações efetivas podem ser estabelecidas, oferecendo assim condições para alcançar o controle metabólico.

Portanto, o profissional de saúde é a pessoa mais indicada para orientar a respeito dos cuidados e pode colaborar na eficácia da adesão ao tratamento, quando associado às suas práticas, ações baseadas nos conhecimentos científicos, mas que também valorizam as crenças, necessidades e especificidades dos clientes $^{(7,19)}$.

Observa-se no depoimento de Margarida que as mudanças mediante a oferta de informações constituem vínculo afetivo por parte das clientes e profissionais, o que possibilita melhor adesão e controle da doença. Sendo assim, enfatizase a importância das orientações profissionais para o cuidado na pessoa com diabetes, se intensificando ainda mais, quando esta pessoa está gestante, devido a todos os riscos decorrentes da não adesão ao plano terapêutico ${ }^{(9)}$.

As gestantes diabéticas devem ter um prénatal regular, com participação efetiva da equipe 
de saúde. Os profissionais precisam estabelecer contratos com os pacientes, promover uma relação de responsabilidade e transparência entre eles, de forma que cada avanço seja parte de um pacto de saúde estabelecido entre ambos.

Elas devem ainda se sentir livres para expressar suas dificuldades, suas insatisfações e para relatar com sinceridade os passos dados e os fatos ocorridos, sejam eles positivos ou negativos, de forma que o profissional tenha sempre uma visão transparente e verdadeira do que acontece com esta mulher. O modo de viver e pensar de cada indivíduo tem influência significativa na adesão do mesmo a qualquer tipo de tratamento ${ }^{(19)}$.

\section{CONSIDERAÇÕES FINAIS}

Quando somada a uma doença crônica como o diabetes, a gravidez passa a ter maior ênfase na vida da mulher, pois necessita manter-se saudável não mais apenas para si, mas também para o filho. Os resultados do estudo nos permitiram verificar que a gestação, considerada como um marco na vida da mulher, atuou como elemento motivador para um melhor cuidado com a doença, favorecendo as mudanças de hábitos de vida, especialmente quanto à alimentação e prática de atividades físicas.

Verificou-se ainda, que a família tem papel fundamental nesta mudança, atuando de forma colaborativa nas mudanças de hábitos diários, especialmente alimentares, melhorando o comportamentodesaúde dasgestantes. As orientações e cuidados em saúde também foram citadas como de fundamental importância para as mudanças.

Sabe-se que a vivência com o diabetes é algo a ser enfrentado dia a dia desde o momento do diagnóstico, contudo, quando conscientes da importância das mudanças, apoiadas pela família, pelos profissionais de saúde que as acompanham, e movidas pela maternidade, estas se tornam mais fortes e confiantes para realizar o autocuidado.

\section{REFERÊNCIAS}

1. Golbert A, Campos MAA. Diabetes melito tipo 1 e gestação. Arq. bras. endocrinol. metabol. 2008;52(2):307-14.

2. Fernandes RSR, Simões AFB, Figueiredo ACN, Ribeiro ARS, Aleixo FMF, Aragüés SMDTG, et al. Pregnancy outcomes in women with pre-existing diabetes. Rev Bras Ginecol Obstet. 2012;34(11):494-8.

3. Lapolla A, Dalfrà MG, Fedele D. Pregnancy complicated by diabetes: what is the best level of HbA1c for conception? Acta diabetol. 2010;47(3):187-92.

4. Persson $M$, Norman $M$, Hanson U. Obstetric and perinatal outcomes in type 1 diabetic pregnancies: a large, population-based study. Diabetes Care. 2009;32(11):2005-9.

5. Rosland AM, Kieffer E, Israel B, Cofield M, Palmisano $\mathrm{G}$, Sinco B, et al. When is social support important? The association of family support and professional support with specific diabetes selfmanagement behaviors. J Gen Intern Med. 2008;23(12):1992-9.

6. Araujo MFM, Pessoa SMF, Damasceno MMC, Zanetti ML. Diabetes gestacional na perspectiva de mulheres gravidas hospitalizadas. Rev. bras. enferm. 2013;66(2):222-7.

7. Petroni LM, Silva TC, Santos AL, Marcon SS, Mathias TAF. Convivendo com a gestante de alto risco: a percepção do familiar. Cienc. cuid saude. 2012;11(3):535-41.

8. Bardin L. Análise do conteúdo. Lisboa: Edições 70; 2011.

9. Cardoso MI, Moraes MAM, Pereira JG. Práticas de autocuidado desenvolvidas por diabéticos tipo $2 \mathrm{em}$ uma unidade de saúde da família. Rev. Eletr. Ge \& S. 2011;2(1):277-90.

10. Moura ERF, Evangelista DR, Damasceno AKC. Conhecimento de mulheres com diabetes mellitus sobre cuidados pré-concepcionais e riscos materno fetais. Rev Esc Enferm USP. 2012;46(1):22-9.

11. Ferreira LE, Zanatta EA, Brumm MLB, Nothaft SCS, Motta MG. Diabetes mellitus sob a ótica do adolescente. Cogitare enferm. 2013;18(1):71-7.

12. Oliveira KCS, Zanetti ML. Conhecimento e atitude de usuários com diabetes mellitus em um serviço de atenção básica à saúde. Rev Esc Enferm USP. [Internet] 2011;45(4) [acesso em 05 jul 2013]. Disponível: http:// dx.doi.org/10.1590/S0080-62342011000400010

13. Oliveira MIV, Bezerra MGA, Filho JGB, Veras MAC, Bezerra JP. Perfil de mães e recém nascidos na presença do diabetes mellitus gestacional. Rev Rene. 2009;10(4):28-36.

14. Nolan JA, McCrone S, Chertok IR. The maternal experience of having diabetes in pregnancy. J Am Acad Nurse Pract. 2011;23(11):611-8.

15. Fonseca CC, Rocha LA. Gestação e atividade física: manutenção do programa de exercícios durante a 
gravidez. Rev. Bras. Cienc. Mov. 2012;20(1):111-21.

16. Figueiredo A, Robalo R, Rocha T, Amaral N. Factores preditores de pré-eclâmpsia na diabetes pré-gestacional. Rev. Port. Diab. 2012;7(4):154-9. Disponível: http://repositorio.chlc.minsaude.pt/ bitstream/10400.17/1010/1/Rev\%20Port\%20Diab\%20 2012\%20154.pdf

17. Beltrame $V$, Brugnerotto $M$, Trentini M, Madureira VSF. A convivência com diabetes mellitus tipo 2. Saude Meio Ambient. 2012;1(1):105-16.

18. Zanetti ML, Biaggi MVB, Santos MA, Péres DS, Teixeira CRS. Atenção à pessoa com diabetes e as repercussões na família. Rev. bras. enferm. 2009;61(2):186-92.

19. Holanda VR, Souza MA, Rodrigues MCPS, Pinheiro AK, Damasceno MMC. Knowledge of pregnant women about gestational diabetes mellitus. J Nurs UFPE on line. 2012;6(7):1648-54. 